\title{
Advances in PTSD Treatment Delivery: Review of Findings and Clinical Considerations for the Use of Telehealth Interventions for PTSD
}

Leslie A. Morland, Psy.D. ${ }^{1,2,3, *}$

Stephanie Y. Wells, Ph.D. ${ }^{4,5}$

Lisa H. Glassman, Ph.D. ${ }^{1}$

Carolyn J. Greene, Ph.D. ${ }^{6,7}$

Julia E. Hoffman, Psy.D. ${ }^{8}$

Craig S. Rosen, Ph.D. ${ }^{9,10}$

\author{
Address \\ ${ }^{*}, 1$ VA San Diego Healthcare System, 3375 La Jolla Village Drive, San Diego, CA, \\ USA \\ Email: Leslie.morland@va.gov \\ ${ }^{2}$ University of California, San Diego, San Diego, USA \\ ${ }^{3}$ Pacific Island Division, National Center for PTSD, Honolulu, HI, USA \\ ${ }^{4}$ Durham VA Health Care System, Durham, NC, USA \\ ${ }^{5}$ VISN 6 Mid-Atlantic MIRECC, Durham, NC, USA \\ ${ }^{6}$ Department of Veterans Affairs Healthcare System, Little Rock, AR, USA \\ ${ }^{7}$ University of Arkansas for Medical Sciences (UAMS), Little Rock, AR, USA \\ ${ }^{8}$ Behavioral Health Strategy, Livongo Health, Inc, Silicon Valley, CA, USA \\ ${ }^{9}$ Dissemination \& Training Division, National Center for PTSD, Palo Alto, CA, USA \\ ${ }^{10}$ Stanford University School of Medicine, Palo Alto, CA, USA
}

Published online: 30 May 2020

(C) This is a U.S. Government work and not under copyright protection in the US; foreign copyright protection may apply 2020

This article is part of the Topical Collection on PTSD

Keywords PTSD · Telehealth · Technology • Videoconferencing

\section{Abstract}

Purpose of review Effective treatments for posttraumatic stress disorder (PTSD) remain underutilized and individuals with PTSD often have difficulty accessing care. Telehealth, particularly clinical videoconferencing (CVT), can overcome barriers to treatment and increase access to care for individuals with PTSD. The purpose of this review is to summarize the literature on the delivery of PTSD treatments through office-based and 
home-based videoconferencing, and outline areas for future research.

Recent findings Evidence-based PTSD treatments delivered through office-based and home-based CVT have been studied in pilot studies, non-randomized trials, and randomized clinical trials. The studies have consistently demonstrated feasibility and acceptability of these modalities as well as significant reduction in PTSD symptoms, non-inferior outcomes, and comparable dropout rates when compared with traditional face-to-face office-based care. Finally, it has been shown that using CVT does not compromise the therapeutic process.

Summary Office-based and home-based CVT can be used to deliver PTSD treatments while retaining efficacy and therapeutic process. The use of these modalities can increase the number of individuals that can access efficacious PTSD care.

\section{Introduction}

Nearly 90\% of American adults will experience a potentially traumatic event in their lives. And while not all trauma survivors will end up meeting the criteria for posttraumatic stress disorder (PTSD), we know that all will be impacted-at least temporarily-by the surprise, fear, helplessness, and horror that accompanies abnormal events like these. Those people who develop a chronic PTSD experience poorer quality of life, impaired functioning, and increased healthcare utilization costs [1-4].

Engagement in and completion of effective treatment for PTSD is of utmost importance to both alleviate suffering and reduce the societal and economic impact of trauma. Evidence-based PTSD treatments, such as cognitive processing therapy (CPT) and prolonged exposure (PE), can substantially lessen PTSD symptoms but engagement in these treatments remains low $[5 \bullet, 6]$, even in the U.S. Department of Veterans Affairs' Healthcare Administration (VA), which has widely implemented CPT and PE. Access to evidence-based PTSD treatments is even lower in community hospitals, clinics, and private practices outside of the VA system. The resolution of individual, social, and economic sequalae of trauma depends on the capacity to systematically identify and dismantle the barriers to care for those with PTSD.

Luckily, there is a deep literature outlining the barriers to be overcome. Individuals with PTSD face several barriers to mental health care such as distance, travel time and cost, privacy concerns, lack of specialty or mental health providers, and perceived stigma [7-9]. When these barriers are coupled with PTSD's characteristic avoidance symptoms, the challenge to receive or engage in effective PTSD treatment may be insurmountable.
Because of these common barriers, many people are unable or prefer alternatives to attend traditional inperson, office-based care. Over the past decade, there has been a shift to utilize telehealth, the use of electronic communications and information technology to provide and support health care when the provider and patient are separated by distance or other limitations (e.g., physical mobility) [10, 11]. Clinical videoconferencing (CVT), one of the most widely studied forms of telemental health, allows a provider and patient in separate locations to meet synchronously through videosharing technology. The patient may be in their home or another private area (home-based CVT) or another healthcare facility (office-based CVT).

The use of CVT for the provision of PTSD services has evolved. Originally, CVT usually utilized a "hub and spoke" model, which allowed a mental health provider located at a centralized "hub" healthcare facility to meet with a patient located at a different healthcare facility closer to their home. This model reduced some of the barriers associated with seeking treatment, such as reduced travel time to a closer facility, and subsequently decreased time and costs [12]. However, office-based CVT still involves some travel time for patients, and does not address other barriers to care, such as privacy concerns about being seen entering a mental health clinic, parking difficulties, and trauma-related cues in clinical settings (e.g., sitting in a crowded waiting room with other patients who may resemble the perpetrator).

As office-based CVT has proved effective and safe, CVT has shifted to occur directly into patients' homes through the use of home-based CVT $[12 \bullet, 13]$. Homebased CVT allows patients to be in the comfort of their 
own homes or another private location, and meet with a provider located at another location, also sometimes their home or non-clinical setting. Therefore, homebased CVT can increase flexibility for both the patient and provider. Home-based CVT eliminates barriers including travel time and cost, privacy concerns, perceived stigma, and logistical challenges such as parking difficulties. Additionally, home-based CVT may increase scheduling flexibility, and if appropriate, include family members to participate in clinical care, which can provide insight into the patient's relationship and home environment. This article will review the current evidence for the use of both office-based and home-based CVT to deliver evidence-based PTSD treatments and outline areas for future research. All studies included in this review will be included in Table 1 .

\section{Evidence for CVT for the delivery of PTSD treatment}

The feasibility and effectiveness of office-based CVT have been established through pilot studies, non-randomized cohort studies, and rigorous randomized non-inferiority trials. Tuerk et al. [14] published the first nonrandomized cohort study examining the use of office-based CVT to provide an evidence-based therapy (EBT) specifically for PTSD with combat veterans. The study included 12 veterans who received PE via telehealth and were compared with a reference sample of 35 veterans who received PE inperson. This study demonstrated significant improvements in PTSD symptoms, as assessed by the PTSD Checklist-Military Version (PCL-M; [15]), at post-treatment following prolonged exposure (PE) therapy delivered via office-based CVT. In a pilot study with 13 male veterans, Morland et al. [16] reported no difference in therapeutic alliance or clinician-assessed PTSD symptoms, as measured by the Clinician-Administered PTSD Scale (CAPS; [17]), between patients receiving CVT or in-person delivery of cognitive processing therapy (CPT) in a group format. These two initial studies provided initial evidence for the feasibility and acceptability of office-based CVT for both individual and group PTSD treatment.

More rigorous randomized trials have tested whether office-based CVT is non-inferior to in-person PTSD treatment. Frueh and colleagues [18] compared group cognitive-behavioral therapy (CBT) for PTSD with group CBT conducted face-to-face in a sample of 38 veterans. The results indicated that there was no significant difference in self-reported PTSD symptoms, as assessed by PCL-M, between group CBT delivered through office-based CVT compared with in-person group CBT at post-treatment and 3-month follow-up. In a study with 125 male veterans receiving group CPT, Morland and colleagues' [19] established that office-based CVT was non-inferior to in-person CPT at post-treatment and 6-month follow-up, with an effect sizes improvement of $d=.78$ and .76 on the CAPS, respectively, in both arms of the study [19]. Office-based CVT was also found to be non-inferior to traditional office-based care for the delivery of individual CPT to female civilians and veterans with PTSD, with a mean improvement of 20.5 points on the CAPS [20]. Maieritsch et al. [21] compared results of CPT delivered via office-based CVT with in-office, in-person individual CPT in a sample of 90 American veterans of the wars in Iraq and Afghanistan. Results from both arms appeared comparable, but the authors suggested that the high 


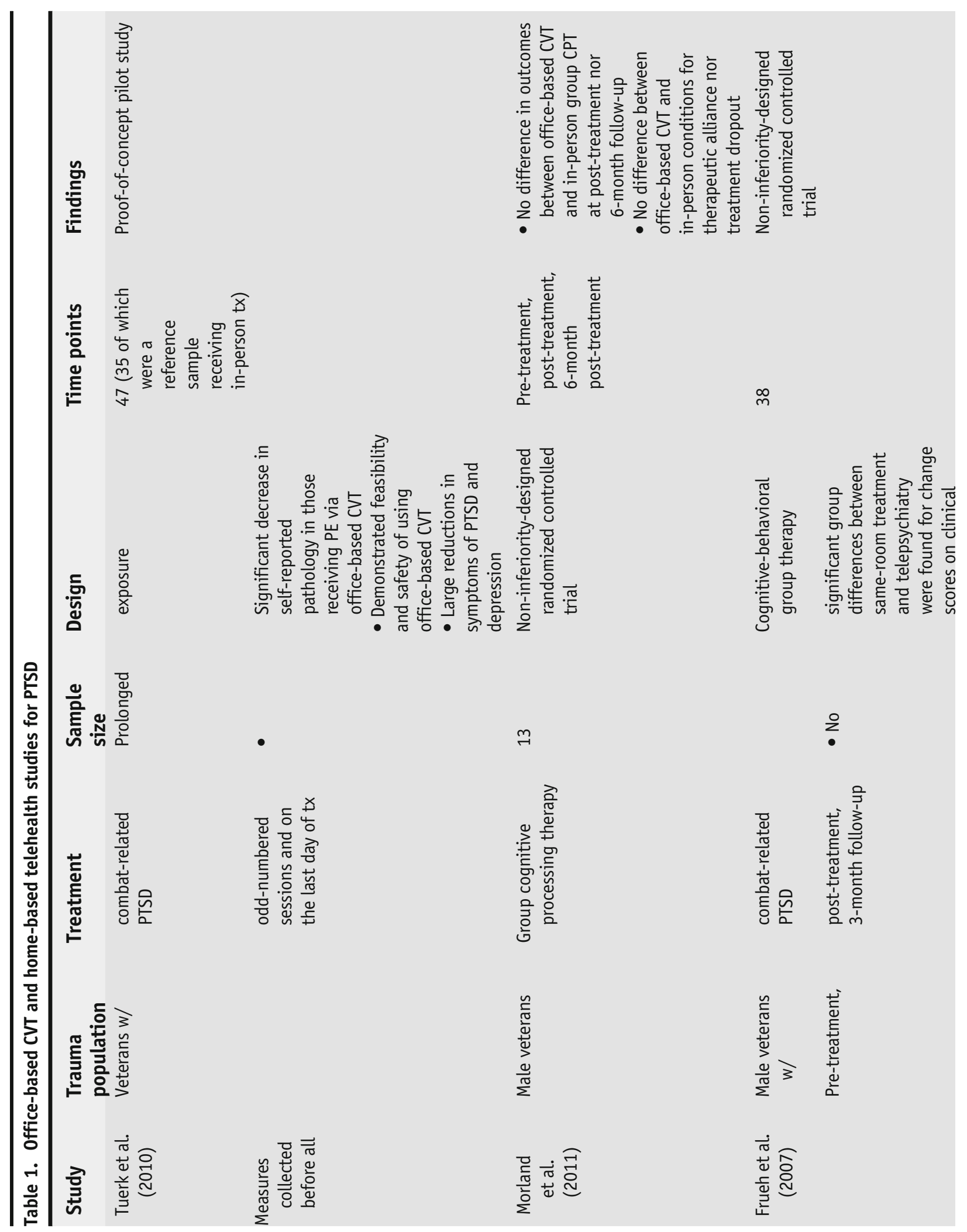




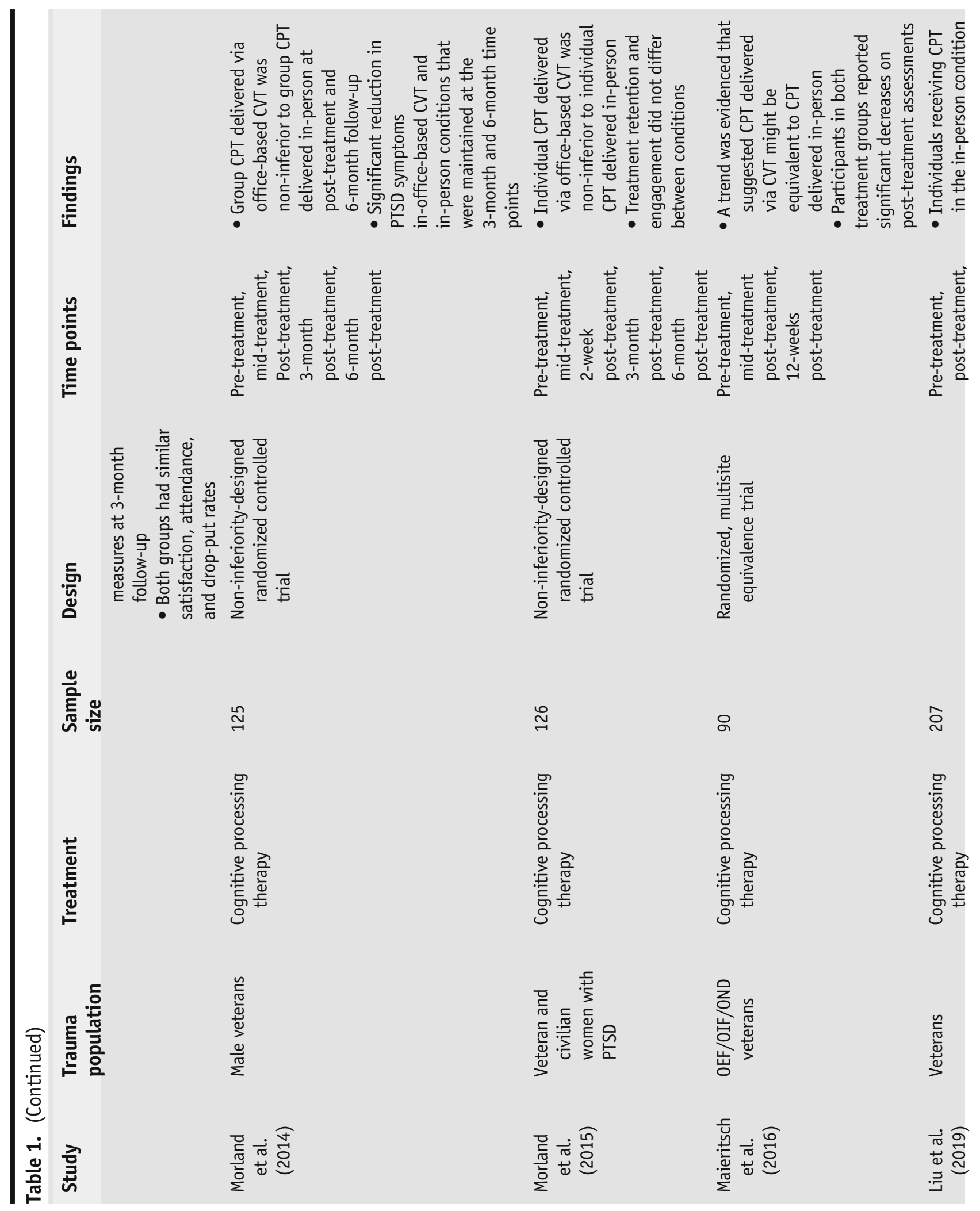




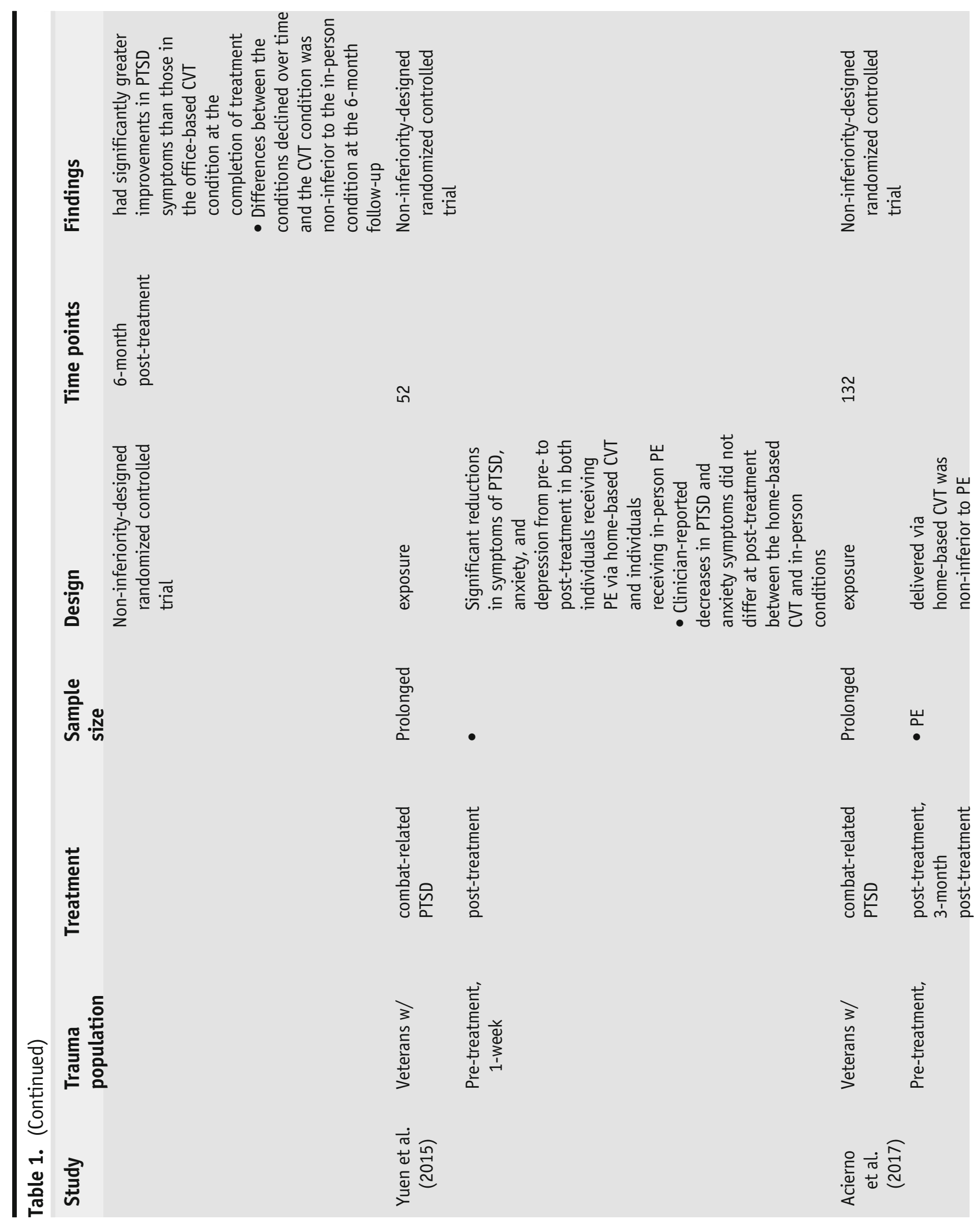




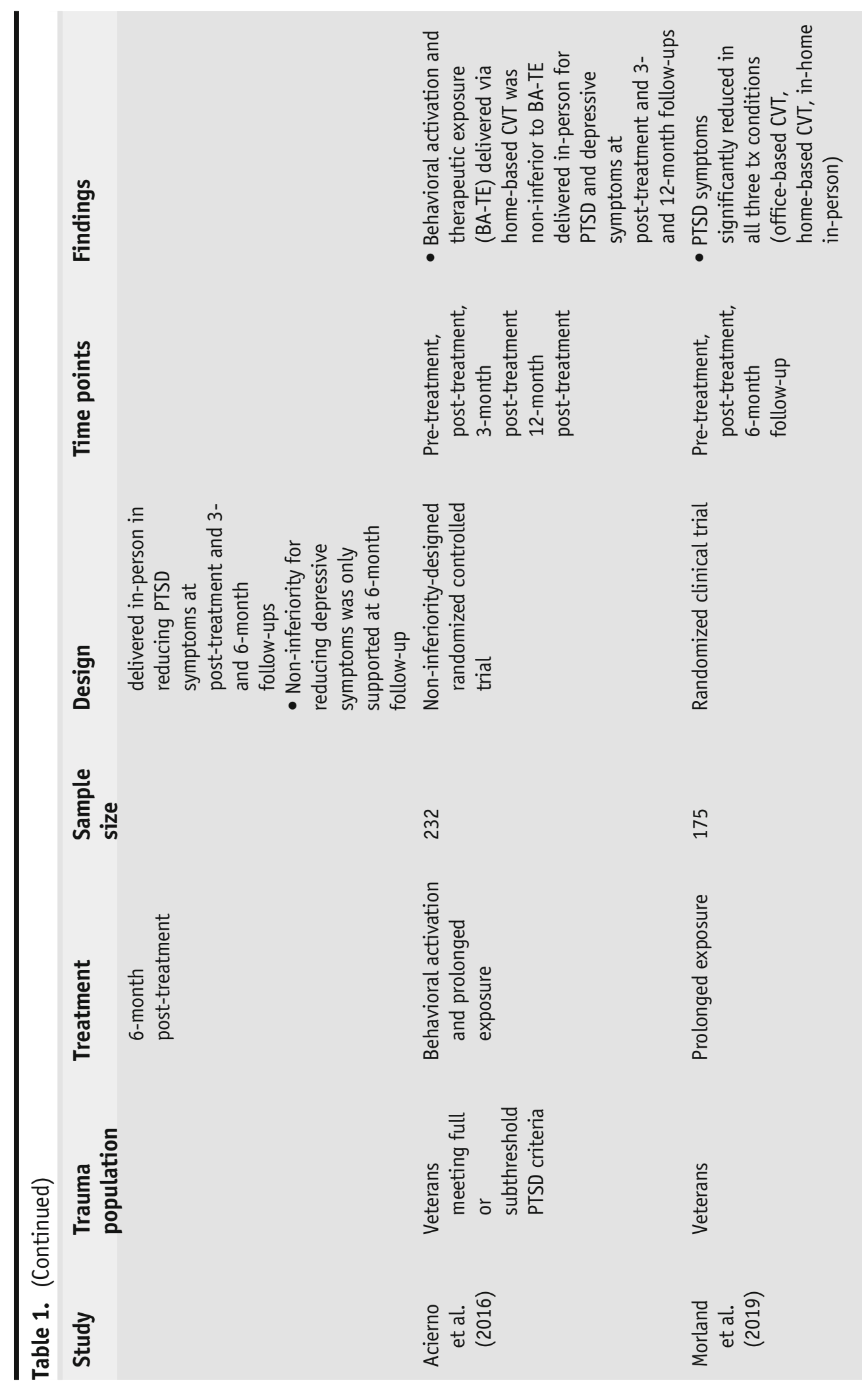


( $43 \%$ across the study) dropout rate from the study prevented the authors from being able to conduct formal non-inferiority analyses. It is possible that the sample size also prevented the authors from being able to establish non-inferiority. There were no significant differences in the dropout rates between the arms, suggesting it was not the modality that impacted veterans' decision to drop out of treatment. The authors also reported that their dropout rates were similar for other studies of veterans who served in Iraq and Afghanistan.

Only one randomized trial [22] suggests a possible difference in PTSD outcomes between in-person psychotherapy and office-based CVT. In a sample of 207 male and female veterans, CAPS scores declined by an average of 19 points among those receiving in-person CPT, but only 9 points among those receiving CPT via office-based CVT. However, the office-based CVT group continued to improve over time, and CVT was noninferior at 6-month follow-up. Overall, the results from these studies indicate that PTSD treatments can be delivered via office-based CVT with effectiveness similar to traditional office-based care.

Following the establishment of the efficacy of office-based CVT, studies began to examine if home-based CVT could yield similar results. Yuen and colleagues [13] published preliminary results that compared PE delivered via home-based CVT to an in-office in-person condition with a group of 52 veterans. PTSD symptoms Clinician-reported PTSD, as assessed by the CAPS, and anxiety symptoms, as assessed by the Beck Anxiety Inventory (BAI; [23]), at post-treatment did not differ between CVT and in-office care. Another study by Acierno and colleagues compared home-based CVT and in-person delivery with a combination of behavioral activation and exposure for PTSD [24]. They used the PCL-M and the Beck Depression Inventory-II (BDI-II; [25]) to determine non-inferiority for PTSD and depressive symptom reduction. CVT was non-inferior to the in-person modality both for PTSD and depression symptoms at post-treatment and 3and 12-month follow-up. A second study by Acierno and colleagues [26] established non-inferiority for PE delivered via home-based telehealth compared with in-person modalities for reducing PTSD symptoms, as assessed by the PCL-M, in a sample of 150 veterans immediately after treatment ( $d=1.24$ improvement from pre-treatment) and 6 months later [26]. This study failed to show that depression outcomes on the BDI-II for the CVT arm were inferior to in-person care at the end of treatment; however, the CVT was non-inferior at 6-month follow-up. Finally, Morland et al. [27] conducted an RCT with 175 male and female veterans and compared office-based CVT, home-based CVT, and in-home, in-person (IHIP) delivery of prolonged exposure therapy (there was no traditional office-based in-person condition.). Results indicated that PTSD symptoms as reported on the PTSD Checklist for DSM-5 [28] and ClinicianAdministered PTSD Scale for DSM-5 [29] significantly reduced following PE delivered via each of the three modalities $(d=.96$ to 1.08$)$ with no difference between treatment arms. These four studies consistently show that exposure therapy for PTSD can be delivered effectively to veterans' 
homes through CVT.

The use of CVT to deliver PTSD treatment has mostly led to results "as good as" in-person care on therapeutic process variables. Most studies have reported comparable attrition between office-based and home-based CVT compared with in-person care $[18,20,24,26]$ indicating that the use of telehealth does not increase dropout rates but also does not decrease dropout. However, one pilot study found that the office-based CVT condition had a slightly higher dropout rate $(25 \%)$ compared with traditional office-based care $(17 \%)$ but the small sample size prevented the authors from being able to conduct inferential statistics [14]. Moreover, the dropout rate (25\%) among the telehealth group was still within the typical range for PTSD treatment [30]. Most recently, Morland and colleagues [27] found comparable attrition rates among individuals who received PE via office-based CVT or home-based CVT. Yet the dropout was lowest when the therapist made an in-person visit to the home rather than conducted the therapy via video. These findings are consistent with a recent CPT study where similar findings demonstrated the lowest dropout rates for homebased therapy options (CVT and in-home in-person) with no differences in treatment efficacy [31]. Thus, the evidence to date suggests that the dropout rates between office-based and home-based telehealth are at least comparable with office-based in-person care, and that in-home CVT may even increase retention because it eliminates travel as a barrier to care. Studies of other process variables suggest that session attendance [18, 32], information retention [33], and patient and clinician satisfaction and alliance [34,35] for CVT are also comparable with in-person care. In summary, the use of telehealth does not appear to compromise the therapeutic process and clinicians can feel confident to utilize telehealth for the provision of care.

Some individuals have expressed concerns that CVT would compromise therapists' adherence to treatment protocols. However, research indicates that the use of CVT does not affect therapist adherence [27,36, 37] or the therapist's ability to maintain treatment fidelity [24] in the delivery of PTSD EBTs. Additionally, therapist competence (i.e., developing rapport, conveying empathy) and adherence (i.e., structuring sessions, providing feedback) to a manualized group CBT for PTSD have been found to be similar in both delivery modalities [18]. Although there is not much specific evidence regarding the use of CVT for non-CBT PTSD interventions, the current findings do suggest that the integrity of specialized treatments, such as CPT and PE, can be maintained over CVT.

Patient-centered care emphasizes the use of patients' preferences in clinical care and patients need to be willing to utilize telehealth for it to be beneficial and increase access to care. Research has shown that individuals with PTSD are willing to receive medical and psychiatric services using a technology modality [38]. Additionally, research has also demonstrated the acceptability [39] and use of telemental health $[40,41]$. However, one study suggested reduced willingness to engage in CVT in a rural population [42].

Patient acceptance of CVT may increase after they first experience therapy via CVT. A recent study conducted among veterans with PTSD living in rural areas examined patient perceptions before and after receiving CPT via home-based CVT using a tablet. Although veterans started the study with neutral or positive 
feelings toward home-based CVT, after treatment, they were notably positive: they preferred it, felt comfortable with it for PTSD treatment, and would recommend it to others [43]. The negative feedback is from a minority of veterans in the study related to safety and privacy concerns or technological problems experienced during CVT therapy that reduced their treatment satisfaction [43]. CVT technology continues to improve rapidly, which may reduce users' negative experiences with CVT but providers should routinely discuss these potential concerns when delivering PTSD treatment through CVT.

As CVT becomes a more established means of offering PTSD treatment, research should move from the acceptability of CVT to examining possible patient preferences for receiving care through telehealth vs. in-person care because patient preferences may vary. In a study of veterans seeking PTSD treatment delivered via home-based teleconferencing, office-based CVT, or in-home in-person care (IHIP), almost half of patients preferred home-based CVT, a third preferred IHIP, and a quarter preferred office-based CVT [26]. Wells and colleagues [44] examined modality preference among 105 veterans seeking couples-based PTSD treatments. Fifty-one percent preferred home-based CVT and $49 \%$ preferred traditional office-based care [44]. Overall, the findings from these two studies suggest that veterans with PTSD are as likely to prefer homebased telehealth as in-person care. Matching patients with their preferred treatment type has been shown to improve clinical outcomes [45]. Future research should examine whether matching individuals with their preferred treatment modality could improve both treatment attendance and outcomes.

\section{Practical and clinical considerations for clinicians}

Expanding providers' practices

Given the feasibility, acceptability, and effectiveness of CVT, providers and healthcare systems may consider implementing CVT to expand the scope of their clinical practice, expand delivery options, increase flexibility, and provide more opportunities to fulfill patients' preferences for how they receive their care. Not only can CVT be used as a standard practice, but it also offers opportunities for clinicians to provide remote care in unique situations, such as when the patient or provider is sick, when a patient is traveling for work, or during a public health crisis, such as a pandemic (e.g., SARS-CoV-2 also known as COVID-19). Although CVT can increase flexibility, it is also important for providers to be trained and comfortable in the use of CVT. In unexpected circumstances, such as the COVID-19 pandemic, there may also be additional considerations. Therefore, we have provided several practical and clinical considerations for providers.

Many individuals with PTSD reside in rural areas and have difficulty accessing specialty providers. Providers may consider using CVT to increase the geographic areas that they serve, thus increasing both their client base and the availability of treatment for individuals in remote locations. In addition to reaching new clientele, CVT can be used to enhance existing patients' care. For example, patients sometimes need to cancel weekly scheduled appointments due to work obligations, travel, or sickness. 
Providers may consider meeting with patients through home-based CVT on an as needed basis to decrease the number of missed appointments due to competing demands. Further, providers may use CVT to continue care for established patients who relocate and would require a referral to a new clinician.

In addition to the videoconferencing platform, providers and patients can extend the therapeutic window through use of messaging software between sessions to ask and answer questions, transmit homework, or identify items to be discussed in the following session. Providers may also decide if they want to offer brief services between sessions through phone or text during the COVID-19 pandemic when patients may be in greater distress. In sum, utilizing CVT and other forms of telehealth can benefit both providers and patients by increasing the number of people who can be served or continuing care when it would otherwise not be possible.

There are several practical and clinical considerations for clinicians conducting therapy via CVT. We outline below several of these and suggested solutions or approaches. However, providers and patients may implement their own solutions that work best for each individual patient.

The use of CVT requires that both patients and providers have access to the necessary equipment, such as a computer, tablet, or smartphone, that can operate videoconferencing technology (e.g., HIPAA compliant versions of Zoom, WebEx, VA Video Connect). Providers that are located in healthcare systems or on grant funded projects may have the opportunity to order equipment to temporarily lend to patients if their own equipment cannot support the platforms. If individuals do not have Wifi to support the platforms, then tablets can be ordered with active data plans, if this is within the provider, project, or healthcare system's budgets. In unique circumstances, such as the COVID-19 pandemic, it will be necessary for patients to have a back-up means of communication, such as a telephone, due to servers' bandwidths being overwhelmed. Providers should discuss this with their patients and inform them that sessions may need to be conducted through the phone. If an individual has a concern about limited phone minutes, then healthcare systems or grants may consider providing pre-paid calling cards to patients who do not have sufficient phone minutes, if it is allowed in the budget. However, in our experience, it is rare for this to be a barrier to telehealth.

There are several methods of getting materials to patients for PTSD treatments. Clinicians can mail out all therapy worksheets (e.g., CPT homework assignments, PE in vivo exposure hierarchy forms) and selfreport measures (e.g., PCL-5, BDI-II) prior to the start of therapy. If providers choose this method, it is helpful to organize all materials in 
a binder organized with dividers labeled for each session. For each session, providers can put the self-report measures, handouts, and worksheets. If a binder is not feasible, then providers can label forms for each session by writing the session number at the top or using a post-it note. This will help patients know which materials to look at for which sessions. During the COVID-19 pandemic, providers may consider printing and mailing documents from their homes without their home addresses to reduce the need to go to the post office due to risk of transmission. However, sending materials through regular mail is often slower and less efficient than online transmission.

If clinicians are unable to mail physical documents then providers can consider sending materials electronically, such as through a secure messaging system, such as VA's My HealtheVet, encrypted email platforms, or they may share files through the CVT platform, if it is an available option (e.g., Zoom). When available, providers may also fax materials to patients if both individuals have a fax machine. If sending materials electronically, it is preferable to have fillable PDFs (e.g., CPT \& PE worksheets, and self-report measures). If this is not possible, then providers can send regular PDFs or Word documents. The VA CPT \& PE coach can also be used to provide patient with the appropriate measures and assignments.

Collecting and reviewing PTSD therapy materials from patients

If providers mailed physical copies of worksheets and self-reports, then veterans may mail them back to the clinician. However, this is more costly and may be less timely than electronic sharing. If both individuals have a fax machine, then they may fax their worksheets to providers. If providers sent hard copies to patients, then patients can fill out the measures and worksheets and then share them with the clinician electronically through several methods. For example, patients can hold each form up to the camera for the therapist to review, read the clinician their answers, take a photo through their phone or tablet, or scan the worksheet and then send it through the CVT platform (e.g., Zoom) or a secure messaging platform (e.g., My HealtheVet). If veterans choose to hold up completed forms up to the screen, then the clinician may screenshot the forms to review in more detail or to store in secure files. Regarding self-report measures, clinicians can also verbally administer these and record answers on a hard copy or a fillable PDF for their records; however, this may increase the time it takes to complete the forms.

If veterans filled out electronic worksheets and self-reports, then they can transfer the materials to the clinician using similar methods, such as secure messaging, file shares through the CVT platform, or sharing their screen with the therapist, which would allow the therapist to take a screenshot. Mobile apps, such as VA's CPT and PE coach, may also be used to complete forms and record and listen to imaginal exposures during PE, which can be used in session and to complete homework assignments rather than using a digital recorder. For providers leading group CPT, it is helpful to ask patients to send their worksheets in advance (e.g., through secure messaging) so that the therapist can review in advance. 
When teaching material to clients (e.g., an $\mathrm{ABC}$ sheet during CPT or an in vivo exposure hierarchy during PE), the provider may use fillable PDFs and share their screen with the patient to model how to do the worksheet and have the patient follow along on their end using a hard copy or electronic copy. Alternatively, they may have the patient fill it out and share the screen with them so that they can provide feedback and ensure accurate completion. Similarly, you may have a patient fill out a hard copy of a form while showing them on a shared screen how to fill out the form if the veteran is unable to access electronic versions of the therapy materials. Clinicians may take notes for certain assignments, such as CPT impact statements, on important points if there is a reason why they cannot obtain the full impact statement from the patient; however, having a completed impact statement is ideal. Providers can still complete all of their necessary forms, such as the PE Therapist Imaginal Exposure Recording Form, either on a hard copy or on a fillable PDF that can be saved in a secure file. Given that servers are often overwhelmed due to increased usage during the COVID-19 pandemic, then providers may need to teach new content over the phone and complete session or part of sessions through the phone. Providers can ask patients to follow along on their worksheets, as usual, and repeat back everything that they write down so that the provider can make their own copy and confirm understanding of the material. Even if the video fails during the session, the provider could ask patients to take a photo of their worksheets through their smartphone or tablet and then send through a secure messaging platform following the completion of a phone session. Unfortunately, providers will be unable to see nonverbal behaviors and facial affect during sessions, such as during an imaginal exposure, and may need to ask more questions afterwards.

CVT platforms may have technological difficulties, such as freezing or disconnected calls, so clinicians should always have an identified back-up method of communication, typically a telephone, for each session. It is helpful for providers to establish this at the beginning of therapy and ask the patient to have the back-up communication available at every session. However, clinicians should ask patients to have their telephones off or on low volume to minimize distractions during session when the back-up communication is not needed. However, in the current COVID-19 pandemic, many providers and patients will need to utilize the telephone to conduct sessions so patients and providers should have their phones ready and nearby to use if the CVT equipment fails. The patient and provider should agree in advance about who will call whom (e.g., the provider will call the patient upon the technology failing). 
provide patients with a document that reviews expectations and boundaries for CVT appointments. Providers may also do this verbally at the first CVT appointment but having a document to refer back to, as needed, can be helpful. Providers should inform patients that they should wear appropriate attire (e.g., the same as they would wear to the hospital or clinic) for appointments. Additionally, it is helpful to inform patients to let providers know if they will be late to a telehealth call, as they would for an in-person appointment. It is also beneficial to inform patients to behave in a similar manner as an in-person appointment, so they should not engage in other activities, such as cooking, texting, emailing, driving, cleaning, smoking, or eating. Individuals may want to have their pets in the room for comfort or convenience. Unfortunately, pets often can serve as safety signals but providers may not be able to see pets in the camera's view (e.g., if they are in their lap). Therefore, providers may ask that they have pets in another room, if possible, and if it is not possible then ask them to try to minimize interaction with pets during appointments. Providers should consider removing personal objects from the camera's view (e.g., family photos) to encourage their own privacy and reduce opportunities for personal questions. Finally, providers may ask patients to stay seated or have the device on a stable surface to reduce motion sickness.

To protect confidentiality, it is important that both the patient and provider have a private location without other individuals or children present. This may be increasingly challenging though given that during the COVID-19 pandemic when many people are working at home, unemployed, caring for children, or sharing space with other individuals who are working from home. If finding a private room is not possible, patients may sit in a parked car or use earphones, if needed. Alternatively, they may ask other individuals in the home to wear earphones so that they cannot overhear the therapy conversations. During the COVID-19 pandemic, providers may consider additional hours (e.g., evening) when individuals may have help with childcare so that they can attend appointments.

Licensure and reimbursement

Laws and regulations change rapidly for providing services across state lines. Thus, providers should be aware of their state's laws. Additionally, not all insurances reimburse for CVT appointments or may not reimburse if the equipment fails and a telephone is used. However, during times of a pandemic such as COVID-19, it is possible that laws and reimbursement requirements may be loosened. It is helpful for providers to try to obtain current information about these topics. The American Psychological Association has useful information about these requirements.

In addition to practical and logistical considerations, the use of CVT has unique clinical considerations. We will outline several of these below with recommendations. 
It is important for clinicians to establish procedures to help ensure patient safety when doing therapy over CVT. Providers should have patients identify an emergency contact, who can be contacted in times of an emergency. This is typically established prior to or during the first appointment and the clinician should have the client sign the appropriate Release of Information (ROI). If mailing an ROI will delay treatment, then providers can obtain this through secure messaging, fax, a photo that is sent through the CVT platform's file share, or the patient can hold it up to the camera and the provider can take a screenshot. Providers may consider contacting the identified emergency contact if the patient were to become severely distressed during a call, hang up, and the provider is unable to reach the patient. Additionally, if a medical emergency were to occur during session, then the provider may contact the emergency contact. The provider should also confirm the client's physical location at the start of every appointment in case they need to contact local emergency personnel if an acute crisis arises and there is a need for an inperson intervention or crisis response. Providers should also document the patient's physical location each session.

There are currently no established contraindications for CVT for individuals with suicidal ideations (SI). However, many individuals with PTSD experience (SI) and trauma-focused treatments can elicit difficult emotions and distress at times. If a client is determined to be moderate or high risk, then providers can still conduct safety plans through CVT and make sure that the patient has a copy. For example, the provider may draft a safety plan on their computer and share their screen with the patient for them to take a screenshot. Alternatively, they could send a copy through secure messaging or the platform's file share, or if needed, they may choose to send a copy in the mail or through fax, if possible. Providers can also review local emergency resources with patients, such as the local emergency room (ER) or psychiatrist on call at a nearby hospital. However, during the COVID-19 pandemic, patients may be concerned about presenting to an ER due to fear of contracting the virus. Therefore, providers should also provide other resources, such as suicide crisis hotlines, and encourage patients to wear personal protective gear (e.g., a mask, gloves) if they do need to go to the ER. Providers may consider offering brief crisis management session through telephone or CVT, as needed, to supplement standard psychotherapy appointments for patients who are at higher risk.

Creating a therapeutic environment is important when conducting therapy through CVT. Patients and providers should both be located where there is adequate lighting so that both individuals can clearly see non-verbals through the screen and so that providers can see worksheets, as needed. However, if a patient is wearing a mask during the COVID-19 pandemic (e.g., if they live with other people who may have been exposed), then they may need to ask more questions about current emotions and allow for longer pauses to allow patients to feel natural emotions. Providers should try to look into the camera, rather than the screen, to facilitate eye contact. 
Also, if using a detachable webcam, patient and provider may need to reangle the camera to optimize the view. Clinicians should have materials readily available, as they would in the office, to facilitate more fluid sessions. It is important that both individuals have a private area to protect confidentiality but as stated earlier this may be increasingly challenging during the COVID-19 pandemic.

\section{Additional assessment}

Providers may need to conduct additional assessment to gather important information because some information can be lost over telehealth. For example, providers may be unable to see the individual's entire body and may be unable to see if they use a wheelchair or have prostheses. Additionally, they may be unable to observe a patient petting their animal for emotional support, which may serve as a safety behavior during distressing content, such as during an imaginal exposure. Providers may need to ask additional questions to confirm the patient's current environment for the session and during an intake they may ask about any concerns about mobility or assistive devices that may not be visible in the camera's view. However, conducting home-based CVT may also offer useful information to the provider about a patient's home environment, which may offer ideas for potential homework assignments (e.g., in vivo exposures).

\section{Future directions}

The current state of the literature demonstrates that CVT is highly effective for delivering PTSD treatment, is preferred by many individuals, and does not compromise the therapeutic process. Nonetheless, challenges still exist to fully maximizing the potential of CVT and additional for areas of future research are warranted. In this section, we will review several areas for future exploration.

Use of CVT for other PTSD treatments

Much of the research on CVT for PTSD has involved delivery of weekly or biweekly CPT or PE, but CVT can also be used to deliver other treatments for trauma survivors. "Massed" variants of PE (10 daily sessions of PE) and СРT (e.g., twice daily sessions for 5 days) have been shown to be effective and can enable recovery in a shorter period of time [46, 47]. However, travel time and time off from work could be major barriers to accessing a daily treatment. Future studies could assess whether CVT, particularly home-based CVT, could be one way of making it easier for people to engage in such intensive treatments. Research is also underway on developing briefer, less resource intensive treatments for PTSD. A recent RCT found a brief five-session PTSD treatment, Written Exposure Therapy (WET; [48]), to be non-inferior to 12 sessions of CPT. Although WET via CVT has not been tested in research, some VA clinicians are experimenting with delivering WET via CVT and are able to use it successfully [49]. Although EMDR 
is among the most effective treatment for PTSD [50•], we are unaware of studies of EMDR delivery via CVT. Remote delivery of EMDR would seem to be feasible with some modifications (e.g. replacing eye movement tracking with tapping), but this has not yet been tested in research. A treatment for couples, cognitive-behavioral conjoint therapy (СВCT; [51]), has been shown to improve PTSD symptoms, interpersonal functioning, and relationship satisfaction. Morland and colleagues [52] are conducting an RCT to examine the efficacy of an 8-session version of CBCT (B-CBCT) compared with PTSD family education, with delivery of B-CBCT being conducted both in-person and via home-based CVT. The findings from this study will inform providers about the provision of B-CBCT through CVT and the unique concerns when delivering couples therapy over CVT (e.g., ongoing assessment of intimate partner violence and safety risk).

CVT may also be effective to deliver treatments for specific symptoms that are common among individuals with PTSD. Anger and sleep are among the top symptoms that veterans with PTSD report wanting to improve during treatment [53]. Morland and colleagues [54] confirmed that group anger management delivered via in-office CVT was non-inferior to in-person group anger management. cognitive-behavioral therapy for insomnia (CBT-I) could also potentially be delivered via CVT. Although CBT-I via CVT has not been tested in a PTSD population, a recent trial among breast cancer patients showed the CBT-I via CVT was more effective than wait list, but not as good as in-person CBT-I [55]. Further, recent evidence supports the efficacy of remote, self-management of insomnia via technology, e.g. apps [56]; however, further study is warranted.

CVT may be used to augment other forms of telehealth, such as apps or online programs. Patients using these tools benefit from having live human support [57]. Although supplemental support is most typically delivered through text or messaging, it could also be delivered via video. For example, CVT offers a way to bolster or augment web-based therapies such as Interapy [58]. While standard Interapy involves therapist-patient communication via text, a therapist could offer the option of adding synchronous video contact in cases where patients are failing to improve as expected or are not meeting their desired treatment goals.

CVT also offers a potential way to step up services when other remote treatments are insufficient. Numerous studies have shown the effectiveness of web-based interventions for PTSD [59], especially when there is support from a coach or counselor to encourage adherence. These coaches are typically unlicensed paraprofessionals or peers. If during this process it becomes apparent that a patient needs more intensive care than the online program can provide, they could be "stepped up" to a licensed provider through CVT contact. That specialist could then assess the patient to determine whether they could benefit from stepping up to evidence-based psychotherapy and/or psychiatric management via CVT. 


\section{Virtual partnerships to expand access to PTSD care}

Access to high quality PTSD care is impacted by the limited number of professionals with the requisite expertise. CVT can be used to connect professionals and organizations to build partnerships between PTSD specialists and local organizations that are serving trauma-exposed populations. Stewart et al. [60] described a school-based program that delivers trauma-focused CBT to African-American teens affected by community violence. The school facilitated referrals and provided a convenient location with a secure CVT connection; remote providers delivered the psychotherapy. This study offers a model that other schools or universities could consider adopting to potentially contract for virtual PTSD specialty services to augment their campus health services. CVT could also be used to expand PTSD care and consultation to settings like prisons, which have limited mental health expertise but high rates of PTSD [61]. CVT could also potentially be used to bolster the mental health services for refugees or other trauma survivors living in countries that have little mental health infrastructure. Local NGOs could use CVT to link their clients to clinicians in other locations who have the necessary language and clinical skills to provide specialized PTSD care. Through such inter-organization collaborations, more individuals with PTSD may be able to access services through CVT.

\section{Conclusion}

PTSD affects many civilians and veterans and has a substantial impact on quality of life and functioning, while also increasing personal and societal economic burden. Fortunately, the use of CVT offers a promising solution to increase access to effective PTSD treatments without compromising the quality of care. Given the numerous studies demonstrating non-inferiority of CVT relative to in-person treatment, psychotherapy via CVT should be an accepted standard of care. Additionally, wider use of CVT would increase treatment delivery options and enable more individuals to be matched with their preferred delivery modality. Research on CVT has demonstrated that this movement toward CVT is not only feasible for patients and providers, but in some cases preferable, and it offers the foundation for scalable solutions to reduce the significant public health impact of PTSD and improve lives.

\section{Compliance with ethical standards}

Conflict of interest

Leslie A. Morland declares that she has no conflict of interest. Stephanie Y. Wells declares that she has no conflict of interest. Lisa H. Glassman declares that she has no conflict of interest. Carolyn J. Greene declares that she has no conflict of interest. Julia E. Hoffman declares that she has no conflict of interest. Craig S. Rosen declares that he has no conflict of interest. 
Human and animal rights and informed consent

All reported studies/experiments with human or animal subjects performed by the authors have been previously published and complied with all applicable ethical standards (including the Helsinki declaration and its amendments, institutional/national research committee standards, and international/national/institutional guidelines).

\section{References and Recommended Reading}

Papers of particular interest, published recently, have been

highlighted as:

- Of importance

$\bullet$ Of major importance

1. Kilpatrick DG, Resnick HS, Milanak ME, Miller MW, Keyes KM, Friedman MJ. National estimates of exposure to traumatic events and PTSD prevalence using DSM-IV and DSM-5 criteria. J Trauma Stress. 2013;26(5):537-47. https://doi.org/10.1002/jts. 21848.

2. McCaslin SE, Maguen S, Metzler T, Bosch J, Neylan TC, Marmar CR. Assessing posttraumatic stress related impairment and well-being: the Posttraumatic Stress Related Functioning Inventory (PRFI). J Psychiatr Res. 2016;72:104-11.

3. Rodriguez P, Holowka DW, Marx BP. Assessment of posttraumatic stress disorder-related functional impairment: a review. J Rehabil Res Dev.

2012;59(5):649-66.

4. Bass E, Golding HLW. The veterans health administrations treatment of PTSD and traumatic brain injury among recent combat veterans [internet]. Washington DC: Congress of the United States, Congressional Budget Office; 2012. p. 52. Available from: https:// www.cbo.gov/sites/default/files/cbofiles/attachments/ 02-09-PTSD.pdf

5.• Maguen S, Li Y, Madden E, Seal KH, Neylan TC, Patterson OV, et al. Factors associated with completing evidence-based psychotherapy for PTSD among veterans in a national healthcaresystem. Psychiatry Res. 2019;274:112-28

A newly published article about completion of PTSD EBPs in a national sample of veterans.

6. Sripada RK, Bohnert KM, Ganoczy D, Pfeiffer PN. Documentation of evidence-based psychotherapy and care quality for PTSD in the Department of Veterans Affairs. Admin Pol Ment Health. 2018;45:353-61.

7. New Freedom Commission on Mental Health. Achieving the promise: transforming mental health care in America. Final report. Rockville, MD: DHHS; 2003. 113 p. Pub. No. SMA-03-3832.

8. Stecker T, Fortney JC, Hamilton F, Ajzen I. An assessment of beliefs about mental health care among veterans who served in Iraq. Psychiatr Serv. 2007;58(10):1358-61.

9. Workforce issues: integrating substance use services into primary care [Internet]. Proceedings; 2011 Aug 10-11; Washington DC. Washington DC: Substance Abuse and Mental Health Services
Administration and Health Resources and Services Administration. Available from: https://www. integration.samhsa.gov/workforce/ondcp_ proceedings_final.pdf

10. Held P, Pollack M. Novel delivery formats of cognitivebehavioral therapies. Psychiatr Ann. 2019;49:336-7.

11. Institute of Medicine (US) Committee on Evaluating Clinical Applications of Telemedicine. In: Field MJ, editor. Telemedicine: a guide to assessing telecommunications in health care. Washington (DC): National Academies Press (US); 1996.

12. Morland LA, Poizner JM, Williams KE, Masino TT, Thorp SR. Home-based clinical video teleconferencing care: clinical considerations and future directions. Int Rev Psychiatry. 2015;27(6):504-12

Provides an overview of home-based CVT including who is appropraite, ethical considerations, and practical suggestions.

13. Yuen EK, Gros DF, Price M, Zeigler S, Tuerk PW, Foa EB, et al. Randomized controlled trial of home-based telehealth versus in-person prolonged exposure for combat-related PTSD in veterans: preliminary results. J Clin Psychol. 2015;71(6):500-12.

14. Tuerk PW, Yoder M, Ruggiero KJ, Gros DF, Acierno R. A pilot study of prolonged exposure therapy for posttraumatic stress disorder delivered via telehealth technology. J Trauma Stress. 2010;23(1):116-23.

15. Weathers F, Litz B, Huska J, Keane T. PTSD checklist-military version. Boston: Nation Center for PTSD. Behavioral Sciences Division; 1994.

16. Morland LA, Hynes AK, Mackintosh M, Resick PA, Chard K. Group cognitive processing therapy for PTSD delivered to rural combat veterans via telemental health: lessons learned from a pilot cohort. J Trauma Stress. 2011;24(4):465-9.

17. Blake DD, Weathers FW, Nagy LM, Kaloupek D, Gusman FDC, Charney DS, Keane TM. ClinicianAdministered PTSD Scale for DSM-IV (CAPS-DX). 1995

18. Frueh BC, Monnier J, Yim E, Grubaugh AL, Hamner MB, Knapp RG. A randomized trial of telepsychiatry for post-traumatic stress disorder. J Telemed Telecare. 2007;13(3):142-7.

19. Morland LA, Mackintosh MA, Greene CJ, Rosen CS, Chard KM, Resick P, et al. Cognitive processing therapy for posttraumatic stress disorder delivered to rural 
veterans via telemental health: a randomized noninferiority clinical trial. J Clin Psychiatry. 2014;75(5):4706.

20. Morland LA, Mackintosh MA, Rosen CS, Willis E, Resick P, Chard K, et al. Telemedicine versus in-person delivery of cognitive processing therapy for women with posttraumatic stress disorder: a randomized noninferiority trial. Depress Anxiety. 2015;32(11):811-20.

21. Maieritsch KP, Smith TL, Hessinger JD, Ahearn EP, Eickhoff JC, Zhao Q. Randomized controlled equivalence trial comparing videoconference and in person delivery of cognitive processing therapy for PTSD. J Telemed Telecare. 2016;22(4):238-43.

22. Liu L, Thorp SR, Moreno L, Wells SY, Glassman LH, Busch AC, et al. Videoconferencing psychotherapy for veterans with PTSD: results from a randomized controlled non-inferiority trial. J Telemed Telecare. 2019:1357633X19853947.

23. Beck AT, Steer RA. Manual for the Beck anxiety inventory. San Antonio: Psychological Corporation; 1990.

24. Acierno R, Gros DF, Ruggiero KJ, Hernandez-Tejada BM, Knapp RG, Lejuez CW, et al. Behavioral activation and therapeutic exposure for posttraumatic stress disorder: a noninferiority trial of treatment delivered in person versus home-based telehealth. Depress Anxiety. 2016;33(5):415-23.

25. Beck AT, Steer RA, Brown GK. Beck depression inventory-II. San Antonio 1996;78(2):490-8.

26. Acierno R, Knapp R, Tuerk P, Gilmore AK, Lejuez C, Ruggiero K, et al. A non-inferiority trial of prolonged exposure for posttraumatic stress disorder: in person versus home-based telehealth. Behav Res Ther. 2017;89:57-65.

27. Morland LA, Mackintosh MA, Glassman LH, Wells SY, Thorp SR, Rauch SA, et al. Home-based delivery of variable length prolonged exposure therapy: a comparison of clinical efficacy between service modalities. Depress Anxiety. 2019:1-10.

28. Weathers FW, Litz BT, Keane TM, Palmieri PA, Marx BP, Schnurr PP. The PTSD Checklist for DSM-5 (PCL-5)Standard [Measurement instrument]. Retrieved from from http://www.ptsd.va.gov. 2013.

29. Weathers FW, Blake DD, Schnurr PP, Kaloupek DG, Marx BP, Keane TM. The clinician-administered PTSD scale for DSM-5 (CAPS-5). Interview available from the National Center for PTSD at www.ptsd.va.gov. 2013 Aug 6;6.

30. Goetter EM, Bui E, Ojserkis RA, Zakarian RJ, Brendel RW, Simon NM. A systematic. Review of dropout from psychotherapy for posttraumatic stress disorder among Iraq and Afghanistan combat veterans. J Trauma Stress. 2015;28(5):401-9.

31. Medellin E, Mintz J, Moring J, Nabity P, Bira L, YoungMcCaughan S, et al. In office, in-home, and telebehavioral health cognitive processing therapy for combat-related PTSD: preliminary results from a randomized clinical trial. Panel presentation given at the San Antonio Military Health System and Universities Research Forum (SURF); 2019 Jun; San Antonio, TX.
32. Shore JH, Manson SM. A developmental model for rural telepsychiatry. Psychiatr Serv. 2005;56(8):97680.

33. Morland LA, Pierce K, Wong MY. Telemedicine and coping skills groups for Pacific Island veterans with post-traumatic stress disorder: a pilot study. J Telemed Telecare. 2004;10(5):286-9.

34. Germain V, Marchand A, Bouchard S, Guay S, Drouin MS. Assessment of the therapeutic alliance in face-toface or videoconference treatment for posttraumatic stress disorder. Cyberpsychol Behav Soc Netw. 2010;13(1):29-35.

35. Gros DF, Lancaster CL, López CM, Acierno R. Treatment satisfaction of home-based telehealth versus inperson delivery of prolonged exposure for combatrelated PTSD in veterans. J Telemed Telecare. 2018;24(1):51-5.

36. Frueh BC, Monnier J, Grubaugh AL, Elhai JD, Yim E, Knapp R. Therapist adherence and competence with manualized cognitive-behavioral therapy for PTSD delivered via videoconferencing technology. Behav Modif. 2007;31(6):856-66.

37. Morland LA, Greene CJ, Grubbs K, Kloezeman K, Mackintosh MA, Rosen C, et al. Therapist adherence to manualized cognitive-behavioral therapy for anger management delivered to veterans with PTSD via videoconferencing. J Clin Psychol. 2011;67(6):629-38.

38. Grubaugh AL, Cain GD, Elhai JD, Patrick SL, Frueh BC. Attitudes toward medical and mental health care delivered via telehealth applications among rural and urban primary care patients. J Nerv Ment Dis. 2008;196(2):166-70.

39. Shore JH, Brooks E, Novins D. In-home monitoring for American Indian veterans with posttraumatic stress disorder. Telemedicine and E-Health. 2008;14:77.

40. Ashwick R, Turgoose D, Murphy D. Exploring the acceptability of delivering cognitive processing therapy (CPT) to UK veterans with PTSD over Skype: a qualitative study. Eur J Psychotraumatol.

2019;10(1):1573128.

41. Shore JH, Brooks E, Anderson H, Bair B, Dailey N, Kaufmann LJ, et al. Characteristics of telemental health service use by American Indian veterans. Psychiatr Serv. 2012;63(2):179-81.

42. Whealin JM, Seibert-Hatalsky LA, Howell JW, Tsai J. Emental health preferences of veterans with and without probable posttraumatic stress disorder. J Rehabil Res Dev. 2015;52(6):725-38.

43. Whealin JM, King L, Shore P, Spira JL. Diverse veterans' pre- and post-intervention perceptions of home telemental health for posttraumatic stress disorder delivered via tablet. Int J Psychiatry Med. 2017;52(1):320.

44. Wells SY, Jaime K, Schnitzer J, Grubbs K, Morland LA. Examining veterans' preferences for family-based posttraumatic stress disorder services. Poster presented at the 34th annual meeting of the International Society for Traumatic Stress Studies. 2018 Nov; Washington, DC. 
45. Zoellner LA, Roy-Byrne PP, Mavissakalian M, Feeny NC. Doubly randomized preference trial of prolonged exposure versus sertraline for treatment of PTSD. Am J Psychiatry. 2019;176(4):287-96.

46. Foa EB, McLean CP, Zang Y, Rosenfield D, Yadin E, Yarvis JS, et al. Effect of prolonged exposure therapy delivered over 2 weeks vs 8 weeks vs present-centered therapy on PTSD symptom severity in military personnel: a randomized clinical trial. JAMA. 2018;319(4):354-64.

47. Held P, Klassen BJ, Small CF, Brennan MB, Van Horn R, Karnik NS, Pollack MH, Zalta AK. A case report of cognitive processing therapy delivered over a single week. cognitive and behavioral practice. 2019.

48. Sloan DM, Marx BP, Lee DJ, Resick PA. A brief exposure-based treatment vs cognitive processing therapy for posttraumatic stress disorder: a randomized noninferiority clinical trial. JAMA Psychiatry. 2018;75(3):233-9.

49. Worley C, Sloan D, Marx B, Wiltsey Stirman S, McGeeVincent P, Rosen C. Written exposure therapy training and implementation pilot. Presentation at Internation Society for Traumatic Stress Studies 35th Annual Meeting; 2019; Bostion, MA.

50. Hamblen JL, Norman SB, Sonis JH, Phelps AJ, Bisson JI, Nunes VD, et al. A guide to guidelines for the treatment of posttraumatic stress disorder in adults: an update. Psychotherapy (Chic). 2019;56(3):359-73 A recent overview of different clinical practice guidelines for PTSD.

51. Monson CM, Fredman SJ. Cognitive-behavioral conjoint therapy for posttraumatic stress disorder: therapist's manual. New York: Guilford Press; 2012.

52. Morland LA, Macdonald A, Grubbs KM, Mackintosh MA, Monson CM, Glassman LH, et al. Design of a randomized superiority trial of a brief couple treatment for PTSD. Contemp Clin Trials Commun. 2019;15:100369.

53. Rosen C, Adler E, Tiet Q. Presenting concerns of veterans entering treatment for posttraumatic stress disorder. J Trauma Stress. 2013;26(5):640-3.

54. Morland LA, Greene CJ, Rosen CS, Foy D, Reilly P, Shore J, et al. Telemedicine for anger management therapy in a rural population of combat veterans with posttraumatic stress disorder: a randomized noninferiority trial. J Clin Psychiatry. 2010;71(7):855-63.

55. Savard MH, Savard J, Simard S, Ivers H. Empirical validation of the Insomnia Severity Index in cancer patients. Psychooncology. 2005;14(6):429-41.

56. Zachariae R, Lyby MS, Ritterband LM, O'Toole MS. Efficacy of internet-delivered cognitive-behavioral therapy for insomnia - a systematic review and metaanalysis of randomized controlled trials. Sleep Med Rev. 2016;30:1-10.

57. Lewis C, Roberts NP, Simon N, Bethell A, Bisson JI. Internet-delivered cognitive behavioural therapy for post-traumatic stress disorder: systematic review and meta-analysis. Acta Psychiatr Scand. 2019;140(6):50821.

58. Lange A, Schrieken B, van de Ven JP, Bredeweg B, Emmelkamp PM, van der Kolk J, et al. "Interapy": the effects of a short protocolled treatment of posttraumatic stress and pathological grief through the Internet. Behav Cogn Psychother. 2000 Apr;28(2):175-92.

59. Morland LA, Greene CJ, Rosen CS, Kuhn E, Hoffman J, Sloan DM. Telehealth and eHealth interventions for posttraumatic stress disorder. Curr Opin Psychol. 2017;14:102-8.

60. Stewart RW, Orengo-Aguayo R, Wallace M, Metzger IW, Rheingold AA. Leveraging technology and cultural adaptations to increase access and engagement among trauma-exposed African American youth: exploratory study of school-based telehealth delivery of traumafocused cognitive behavioral therapy. J Interpers Violence. 2019:886260519831380.

61. Baranyi G, Cassidy M, Fazel S, Priebe S, Mundt AP. Prevalence of posttraumatic stress disorder in prisoners. Epidemiol Rev. 2018;40(1):134-45.

\section{Publisher's Note}

Springer Nature remains neutral with regard to jurisdictional claims in published maps and institutional affiliations. 